\title{
Cross section of a resonant-mass detector for scalar gravitational waves
}

\author{
M. Bianchi, ${ }^{1,2}$ M. Brunetti, ${ }^{1,2}$ E. Coccia,,${ }^{1,2}$ F. Fucito, ${ }^{2}$ and J. A. Lobo ${ }^{3}$ \\ ${ }^{1}$ Dipartimento di Fisica, Università di Roma “Tor Vergata,'” Rome, Italy \\ ${ }^{2}$ I.N.F.N. Sezione di Roma II, Via della Ricerca Scientifica, 00133 Roma, Italy \\ ${ }^{3}$ Departament de Fisica Fonamental, Universitat de Barcelona, Diagonal 647, Barcelona, Spain
}

(Received 19 September 1997; published 23 March 1998)

\begin{abstract}
Gravitationally coupled scalar fields, originally introduced by Jordan, Brans and Dicke to account for a non-constant gravitational coupling, are a prediction of many non-Einsteinian theories of gravity not excluding perturbative formulations of string theory. In this paper, we compute the cross sections for scattering and absorption of scalar and tensor gravitational waves by a resonant-mass detector in the framework of the Jordan-Brans-Dicke theory. The results are then specialized to the case of a detector of spherical shape and shown to reproduce those obtained in general relativity in a certain limit. Eventually we discuss the potential detectability of scalar waves emitted in a spherically symmetric gravitational collapse.
\end{abstract}

[S0556-2821(98)04908-X]

PACS number(s): 04.80.Cc, 04.30.Db

\section{INTRODUCTION}

Possible extensions of Einstein's theory of gravitation to include scalar fields have received much attention in the past years. The existence of a scalar component in the gravitational field was been originally proposed by Jordan and independently by Brans and Dicke [1] in order to devise a theoretical framework allowing for variations of the fundamental constants and violations of the (strong) equivalence principle. Many other non-Einsteinian theories of gravity incorporate scalar fields. Most notably, string theory, the most serious candidate for a fully consistent quantum theory of gravity, generically predicts the existence of neutral scalar fields [2]. In particular all perturbative string vacua include a scalar, known as the dilaton, in their massless spectrum. Its vacuum expectation value, which plays the role of the string coupling constant, is neither fixed at the classical level nor at any order in perturbation theory. Very poorly understood non-perturbative effects may generate a potential for the dilaton and the other scalar fields, thus stabilizing their expectation value. This mechanism is by no way incompatible with some scalars remaining massless [3]. Active research in this field gives new motivations for further investigating theories of gravitation including other scalars (dilaton, axions or the superpartners of the known fermions). In this respect, it is worth observing that, assuming the validity of a least coupling principle [3], the subsector of string theory determining the coupling of the dilaton has the same functional form as the Jordan-Brans-Dicke (JBD) theory.

Most probably, the existence of massless gravitationally coupled scalar fields would be detected through deviations from general relativity (GR) in the spin contents of gravitational waves (GWs). In this respect, one of the most promising sources of GWs is given by the gravitational collapse of a star [4]. Since in GR no gravitational radiation is emitted in a spherically symmetric collapse, most of the existing literature focuses on the non-spherically symmetric case which generates GWs of spin two. However, in scalar-tensor theories, scalar gravitational waves are radiated from a spheri- cally symmetric collapse. In the Oppenheimer-Snyder approximation, such an emission process has been recently studied [5]. Theoretical predictions for the amplitude of GWs depend on the specific model chosen to describe the collapse and on the assumed theory of gravity. The only constraint is that the assumed theory of gravity must agree, in the regime of weak gravitational fields, with the existing experimental data [6] which support GR to a high degree of accuracy. In the regime of strong gravitational fields the situation is different and large deviations from GR are possible in principle [7]. Eventually, we will argue that scalar GWs emitted in a spherically symmetric collapse in the strong field regime could give a measurable effect for a source within our local group of galaxies.

Among the GW detectors which are now under study or in construction, those with spherical symmetry [8-11] are in a priviledged position $[12,13]$ to detect and discriminate scalar waves. Neither a single cylindrical-shape resonant-mass detector nor a single laser interferometer are in fact able to perform this task. A proposed omnidirectional observatory made out of six cylindrical resonant-mass detectors [14] should be able to discriminate the scalar component of a $\mathrm{GW}$. A minimum of four laser interferometers are needed to discriminate the scalar mode [15]. On the contrary a single spherical resonant-mass detector was shown to be able to detect and discriminate a scalar mode $[13,16]$ and to act as a veto for different theories of gravity [17]. This can be accomplished by monitoring the five degenerate fundamental quadrupole modes of vibration of the detector together with the fundamental monopole mode. In fact, in any metric theory of gravity the "electric" component of the Riemann tensor $R_{0 i 0 j}$ can be written (in the so-called Jordan-Fierz frame) as

$$
R_{0 i 0 j}=S_{i j}+\frac{1}{3} T \delta_{i j}
$$

where $S_{i j}$ is a traceless symmetric tensor, and $T=R_{0 i 0 i}$ is the 
trace part. ${ }^{1}$ From measurements of the above (quadrupole and monopole) modes one is able to completely reconstruct $R_{0 i 0 j}[16,17]$.

In order to make quantitative predictions about the possibility of detecting scalar GWs, we compute in this paper the cross section for scattering and absorption of scalar and tensor GWs by a spherical resonant-mass detector in the framework of the JBD theory. We then apply the results to estimate the potential ability of such a GW detector to sense the characteristic signal emitted in the process of a spherically symmetric stellar collapse.

\section{SCALAR AND TENSOR GWS IN THE JBD THEORY}

Scalar particles may be coupled to gravity in many ways consistent with general covariance. Experimental tests of the equivalence principle, however, put severe constraints and tend to favor theories that predict a universal coupling of such scalar particles to the rest of matter fields [3]. For a single scalar, which may be thought of as the string dilaton, the relevant couplings may be encoded in the JBD theory. In the Jordan-Fierz frame, in which the scalar mixes with the metric but decouples from matter, the action reads

$$
\begin{aligned}
S= & S_{\text {grav }}\left[\phi, g_{\mu \nu}\right]+S_{\mathrm{m}}\left[\psi_{m}, g_{\mu \nu}\right] \\
= & \frac{c^{3}}{16 \pi} \int d^{4} x \sqrt{-g}\left[\phi R-\frac{\omega_{B D}}{\phi} g^{\mu \nu} \partial_{\mu} \phi \partial_{\nu} \phi\right] \\
& +\frac{1}{c} \int d^{4} x L_{\mathrm{m}}\left[\psi_{\mathrm{m}}, g_{\mu \nu}\right],
\end{aligned}
$$

where $\omega_{B D}$ is a dimensionless constant, whose lower bound is fixed to be $\omega_{B D} \approx 600$ by experimental data [18], $g_{\mu \nu}$ is the metric, $\phi$ is a scalar field, and $\psi_{\mathrm{m}}$ collectively denotes the matter fields of the theory. The part of the Lagrangian which describes the matter sector does not depend on the field $\phi$, and it is the same as in GR. Notice that a Weyl rescaling of the metric, $g_{\mu \nu}=\phi^{-1 / 2} g_{\mu \nu}^{E}$, brings the gravitational part of the action to the standard Einstein-Hilbert form but introduces direct couplings of the scalar field $\phi$ to matter. In order to perform our computations the Jordan-Fierz frame proves to be more convenient than Einstein's. The independence of the physical results on the frame choice can be explicitly checked.

As a preliminary analysis, we perform a weak field approximation around the Minkowskian metric and a constant expectation value for the scalar field

$$
\begin{gathered}
g_{\mu \nu}=\eta_{\mu \nu}+h_{\mu \nu} \\
\phi=\phi_{0}+\xi .
\end{gathered}
$$

The standard parametrization $\phi_{0}=2\left(\omega_{B D}+2\right) / G\left(2 \omega_{B D}\right.$ +3 ), with $G$ the Newton constant, reproduces GR in the limit $\omega_{B D} \rightarrow \infty$ which implies $\phi_{0} \rightarrow 1 / G$. The linearized field

\footnotetext{
${ }^{1} \mathrm{~A}$ convenient basis for symmetric rank two tensors is $\left\{S_{(2 m)}^{i j} ; S_{(00)}^{i j}\right\}[16,17]$, which allows one to express the spherical harmonics with $l=0$ and $l=2$ as $Y_{(l m)}=S_{(l m)}^{i j} \hat{x}_{i} \hat{x}_{j}$.
}

equations which correspond to the variation of Eq. (2.1) with respect to $g_{\mu \nu}$ are then given by

$$
\begin{aligned}
& -\frac{1}{2}\left(\partial_{\alpha} \partial^{\alpha} h_{\mu \nu}-\partial^{\alpha} \partial_{(\mu} h_{\nu) \alpha}+\partial_{\mu} \partial_{\nu} h\right) \\
& \quad+\frac{1}{2} \eta_{\mu \nu}\left(\partial_{\alpha} \partial^{\alpha} h-\partial^{\alpha} \partial^{\beta} h_{\beta \alpha}\right) \\
& =\frac{8 \pi}{c^{4} \phi_{0}} T_{\mu \nu}+\frac{1}{\phi_{0}}\left[\partial_{\mu} \partial_{\nu} \xi-\eta_{\mu \nu} \partial_{\alpha} \partial^{\alpha} \xi\right]
\end{aligned}
$$

where $h$ is the trace of the fluctuation $h_{\mu \nu}$ and $T_{\mu \nu}$ is the matter stress-energy tensor. Defining the new field

$$
\theta_{\mu \nu}=h_{\mu \nu}-\frac{1}{2} \eta_{\mu \nu} h-\eta_{\mu \nu} \frac{\xi}{\phi_{0}}
$$

and choosing the gauge

$$
\partial_{\mu} \theta^{\mu \nu}=0
$$

yields the final form of the linearized field equations:

$$
\begin{aligned}
\partial_{\alpha} \partial^{\alpha} \theta_{\mu \nu} & =-\frac{16 \pi}{\phi_{0}} T_{\mu \nu}, \\
\partial_{\alpha} \partial^{\alpha} \xi & =\frac{8 \pi}{2 \omega_{B D}+3} T
\end{aligned}
$$

Far from the sources these equations admit wave-like solutions

$$
\begin{aligned}
\theta_{\mu \nu}(x) & =A_{\mu \nu}(\vec{x}, \omega) \exp \left(i k^{\alpha} x_{\alpha}\right)+\text { c.c. } \\
\xi(x) & =B(\vec{x}, \omega) \exp \left(i k^{\alpha} x_{\alpha}\right)+\text { c.c. }
\end{aligned}
$$

Without affecting the gauge condition (2.5), we can impose $h=-2 \xi / \phi_{0}$ (so that $\theta_{\mu \nu}=h_{\mu \nu}$ ). Gauging away the superflous components, we can write the amplitude $A_{\mu \nu}$ in terms of the three degrees of freedom associated with states with helicities $\pm 2,0[20]$. For a wave travelling in the $z$-direction, we thus obtain

$$
A_{\mu \nu}=\left(\begin{array}{cccc}
0 & 0 & 0 & 0 \\
0 & e_{11}-b & e_{12} & 0 \\
0 & e_{12} & -e_{11}-b & 0 \\
0 & 0 & 0 & 0
\end{array}\right),
$$

where $b=B / \phi_{0}$.

\section{CROSS SECTIONS FOR RESONANT MASS DETECTORS}

Before performing the computation of the cross section we would like to clearly state the nature of our approximations. We consider GWs emitted from a distant source. For the purposes of our computations, we are not interested in the details of the emission but we assume that the GW has the form given in Eqs. (2.8), (2.9) with a frequency $\omega$ $=c|\vec{k}|$ coincident with one of the vibrational eigenfrequen- 
cies of the detector. When the GW impinges on the resonantmass detector, a part of the GW gets scattered and the rest is absorbed. The size of the detector, $R$, is such that $R \ll \lambda$, where $\lambda$ is the GW wavelength, so that the interaction is point-like ("quadrupole approximation'). Once excited, the detector re-emits part of the absorbed radiation, while the rest is transformed into noise.

In the following we compute the scattering cross section

$$
\sigma_{\text {scat }}:=\frac{P_{\text {scat }}}{\Phi}
$$

where $\Phi$ is the incident GW energy per unit time and unit area, and $P_{\text {scat }}$ is the power subtracted by the scattered wave, and the total cross section:

$$
\sigma_{t o t}:=\frac{P_{s c a t}+P_{a b s}}{\Phi}=-\frac{P_{i n t}}{\Phi},
$$

where $P_{a b s}$ is the power absorbed by the detector and $P_{i n t}$ is the power associated to the interference between the incident and the scattered wave [19].

All the computations will be performed twice: once for the tensorial waves (2.8) and once for the scalar waves (2.9). We note that the GW given by Eq. (2.8) receives contributions both from a traceless tensor term and from a scalar term, the trace of $\theta_{\mu \nu}$. The computation of the traceless part is similar to the one performed in the context of standard $\mathrm{GR}$, which is recovered in the limit $\omega_{B D} \rightarrow \infty$. Since the computation in the latter case is very well-known, we will parallel it, describing the general framework but omitting some details which can be found in [19]. All the formalism developed will then be applied to the scalar case.

At large distances from the detector, $r=|\vec{x}| \rightarrow \infty$, the GW is a superposition of a plane wave and a scattered wave:

$$
\theta_{\mu \nu}(\vec{x}, t) \rightarrow\left[A_{\mu \nu} e^{i k \cdot x}+H_{\mu \nu}(\hat{x}) \frac{e^{i \omega r}}{r}\right] e^{-i \omega t}+\text { c.c. }
$$

where $H_{\mu \nu}$ is the scattering amplitude. Expanding the plane wave in spherical waves, we get

$$
e^{i k \cdot x} \rightarrow \frac{e^{i \omega r}}{i \omega r} \delta(1-\hat{k} \cdot \hat{x})-\frac{e^{-i \omega r}}{i \omega r} \delta(1+\hat{k} \cdot \hat{x}),
$$

where $\hat{k}$ and $\hat{x}$ are the unit vectors in the direction of $\vec{k}$ and $\vec{x}$ respectively. Plugging Eq. (3.4) back into Eq. (3.3) yields

$$
\theta_{\mu \nu} \rightarrow\left[A_{\mu \nu}^{\text {out }} e^{i \omega r}+A_{\mu \nu}^{\text {in }} e^{-i \omega r}\right] e^{-i \omega t}+\text { c.c. },
$$

where

$$
\begin{aligned}
& A_{\mu \nu}^{\text {out }}(\vec{x})=\frac{1}{i \omega r}\left[A_{\mu \nu} \delta(1-\hat{k} \cdot \hat{x})+i \omega H_{\mu \nu}(\hat{x})\right], \\
& A_{\mu \nu}^{\text {in }}(\vec{x})=-\frac{1}{i \omega r} A_{\mu \nu} \delta(1+\hat{k} \cdot \hat{x}) .
\end{aligned}
$$

If we choose a GW travelling in the $z$ direction, with wave vector $k^{\mu}=(\omega, 0,0, \omega)$, the perturbation $\theta_{\mu \nu}$ will have non-vanishing components only for $i, j \neq 0, z$ [see Eq. (2.10)].
Then, keeping into account this choice, we can introduce the stress-energy pseudo-tensor $t_{\mu \nu}$, which results from an expansion of the equations of motion to second order in the weak fields. In particular the mixed components read

$$
\begin{aligned}
\left\langle t_{0 z}\right\rangle= & -\hat{z} \frac{\phi_{0} c^{4}}{32 \pi}\left[\frac{4\left(\omega_{B D}+1\right)}{\phi_{0}^{2}}\left\langle\left(\partial_{0} \xi\right)\left(\partial_{0} \xi\right)\right\rangle\right. \\
& \left.+\left\langle\left(\partial_{0} h_{\alpha \beta}\right)\left(\partial_{0} h^{\alpha \beta}\right)\right\rangle\right],
\end{aligned}
$$

where the symbol $\langle\cdots\rangle$ implies an average over a region of size much larger than the wavelength of the GW. Substituting Eqs. (2.8), (2.9) into Eq. (3.8) we get

$$
\begin{aligned}
\left\langle t_{0 z}\right\rangle= & -\hat{z} \frac{\phi_{0} c^{4} \omega^{2}}{16 \pi}\left[\frac{2\left(2 \omega_{B D}+3\right)}{\phi_{0}^{2}}|B|^{2}+A^{\alpha \beta *} A_{\alpha \beta}\right. \\
& \left.-\frac{1}{2}\left|A_{\alpha}^{\alpha}\right|^{2}\right]
\end{aligned}
$$

and using Eq. (2.10), we obtain

$$
\left\langle t_{0 z}\right\rangle=-\hat{z} \frac{\phi_{0} c^{4} \omega^{2}}{8 \pi}\left[\left|e_{11}\right|^{2}+\left|e_{12}\right|^{2}+\left(2 \omega_{B D}+3\right)|b|^{2}\right] .
$$

From Eq. (3.10) we see that the purely scalar contribution, associated with $b$, and the traceless tensorial contribution, associated with $e_{\mu \nu}$, are completely decoupled and can thus be treated independently.

\section{A. Cross section for tensor GWs}

For spin-two waves, the scattering cross section is given by $[19]$

$$
\sigma_{\text {scat }}=\frac{\int\left[f^{\lambda \nu *} f_{\lambda \nu}-\frac{1}{2}\left|f_{\alpha}^{\alpha}\right|^{2}\right] d \Omega}{\left[e^{\lambda \nu *} e_{\lambda \nu}-\frac{1}{2}\left|e_{\alpha}^{\alpha}\right|^{2}\right]},
$$

where $f_{\mu \nu}$ is the spin-two component of the scattering amplitude $H_{\mu \nu}$. The total cross section is given by [19]

$$
\sigma_{\text {tot }}=\frac{4 \pi \mathfrak{I}\left\{e^{\lambda \nu *} f_{\lambda \nu}(\hat{k})-\frac{1}{2} e^{\beta *}{ }_{\beta} f_{\alpha}^{\alpha}(\hat{k})\right\}}{\omega\left[e^{\lambda \nu *} e_{\lambda \nu}-\left.\frac{1}{2}\left|e^{\alpha}\right|^{2}\right|^{2}\right]} .
$$

The ratio between the two,

$$
\eta=\frac{\sigma_{\text {scat }}}{\sigma_{\text {tot }}}
$$

exactly coincides with the ratio between the energy reemitted from the resonant detector as GWs and the energy transformed into noise, viz.,

$$
\eta=\frac{1}{\Gamma} \frac{P_{G W}}{E_{o s c}},
$$

where $1 / \Gamma$ is the decay time of the free oscillation of the detector. 
Which particular combination of vibrational modes of the detector gets excited clearly depends on the polarization of the incoming GW. In the "quadrupole approximation" the scattering amplitude can be expressed as

$$
f_{\mu \nu}(\hat{x})=\tau_{\mu \nu}(\hat{x})-\frac{1}{2} \eta_{\mu \nu} \tau(\hat{x}),
$$

where $\tau_{\mu \nu}(\hat{x})$ are (proportional to) the Fourier transform of the stress-energy tensor at $\vec{k}^{\prime}=\omega \hat{x}$. Stress-energy conservation allows one to recast Eq. (3.11) into

$$
\sigma_{t o t}=\frac{2 \pi \mathfrak{F}\left\{e_{11}^{*}\left(\tau_{11}-\tau_{22}\right)+2 e_{12}^{*} \tau_{12}\right\}}{\omega\left[\left|e_{11}\right|^{2}+\left|e_{12}\right|^{2}\right]},
$$

which depends only on the traceless components of $e_{\mu \nu}$ and $\tau_{\mu \nu}$, and Eq. (3.12) into

$$
\sigma_{\text {scat }}=\frac{4 \pi\left[\tau_{i k}^{*} \tau_{i k}-\frac{1}{3}\left|\tau_{i i}\right|^{2}\right]}{5\left[\left|e_{11}\right|^{2}+\left|e_{12}\right|^{2}\right]} .
$$

For a "pointlike" spherical detector $(R \ll \lambda)$,

$$
\tau_{j k}(\omega)=\gamma e_{j k}
$$

where $\gamma$ is a constant to be determined shortly. By introducing a set of five matrices $S_{(2 m)}^{j k}$ which form a convenient basis for the spherical harmonics with $l=2$ [16], and choosing $+z$ as the direction of propagation, we obtain

$$
\begin{aligned}
\tau^{j k}(\omega)= & \gamma \sqrt{\frac{8 \pi}{15}}\left[e_{11}\left(S_{(22)}^{j k}+S_{(2-2)}^{j k}\right)\right. \\
& \left.-i e_{12}\left(S_{(22)}^{j k}-S_{(2-2)}^{j k}\right)\right] .
\end{aligned}
$$

From Eq. (3.19), it is clear that a tensorial GW propagating in the $+z$-direction will excite only two modes of the detector, precisely those with $l=2$ and $m= \pm 2$. Plugging Eq. (3.18) into Eqs. (3.16) and (3.17), the condition (3.13) becomes

$$
\mathfrak{F}(\gamma)=\frac{2 \omega}{5 \eta}|\gamma|^{2},
$$

which allows us to determine $\gamma$ in terms of $\eta$ and $\omega$. Moreover, at resonance $\left(\omega \simeq \omega_{2}\right)$ the modes of $T^{\mu \nu}$ behave as damped harmonic oscillators. Fourier transforming, one easily infers the $\omega$-dependence of $\tau^{\mu \nu}$, and finally gets

$$
\sigma_{t o t}=\left(\frac{10 \pi \eta c^{2}}{\omega^{2}}\right) \frac{\Gamma^{2} / 4}{\left(\omega-\omega_{2}\right)^{2}+\Gamma^{2} / 4}
$$

where $\omega_{2}$ is the resonance frequency of one of the quadrupole modes $(l=2)$ of the detector. The eigenfrequencies $\omega_{n l}$ can be simply labeled by the radial quantum number $n$ and the principal angular quantum number $l$, since by spherical symmetry they do not depend on the azimuthal quantum number $m$, i.e., they are $(2 l+1)$-fold degenerate. A few numerical values of the eigenfrequencies $\omega_{n l}$ of the spheroi- dal modes can be found, for example, in $[16,17] .^{2}$ The last task we have to perform is the computation of $\eta$ from Eq. (3.14). To this end we need the power emitted as GWs from the detector. For the spin-two components under consideration,

$$
P_{G W}=\frac{2 \omega^{6}}{5 \phi_{0} c^{5}} D_{(T)}^{i j *}(\omega) D_{i j(T)}(\omega)
$$

The traceless quadrupole moment is defined as

$$
D_{(T)}^{i j}(\omega)=D^{i j}(\omega)-\frac{1}{3} \delta^{i j} D_{k}^{k}(\omega),
$$

where

$$
D^{i j}(\omega):=\int x^{i} x^{j} \rho(\vec{x}, \omega) d \vec{x}
$$

is the quadrupole moment of the detector and $\rho$ is the mass density. This quadrupole moment is due to the mass variation of the detector forced by the incoming GW. Its computation is not particularly enlightening, and we report here only the final result for a spherical detector of radius $R$ :

$$
\begin{aligned}
D_{(T)}^{i j}(t)= & \frac{16 \pi}{15} \rho R^{4} C(n, 2) e^{-i \omega_{n 2} t} \sum_{m} S_{(2 m)}^{i j} \\
& \times\left[\beta_{3}\left(k_{n 2} R\right) \frac{j_{2}\left(q_{n 2} R\right)}{q_{n 2} R}\right. \\
& \left.-3 \frac{q_{n 2}}{k_{n 2}} \beta_{1}\left(q_{n 2} R\right) \frac{j_{2}\left(k_{n 2} R\right)}{k_{n 2} R}\right]+ \text { c.c. } \\
= & D^{i j}(t)+\frac{8 \pi}{3} \rho R^{4} C(n, 0) e^{-i \omega_{n 0}{ }^{t}} S_{(00)}^{i j} \\
& \times\left[\beta_{3}\left(k_{n 0} R\right) \frac{j_{2}\left(q_{n 0} R\right)}{q_{n 0} R}\right]+\text { c.c. },
\end{aligned}
$$

where $j_{l}(x)$ are spherical Bessel functions [21], and

$$
q_{n l}^{2}=\frac{\rho \omega_{n l}^{2}}{\lambda+2 \mu} ; \quad k_{n l}=\frac{\rho \omega_{n l}^{2}}{\mu}
$$

enforce the dependence on the material used to build the detector through the Lamé coefficients $\lambda, \mu$. The auxiliary functions $\beta_{i}$ 's are

$$
\begin{aligned}
& \beta_{1}(z):=\frac{d}{d z}\left(\frac{j_{l}(z)}{z}\right) \\
& \beta_{2}(z):=\frac{d^{2} j_{l}(z)}{d z^{2}}
\end{aligned}
$$

\footnotetext{
${ }^{2}$ Toroidal modes of a spherical detector cannot be excited by GWs in any metric theory, and can thus be used as a veto in the detection [17].
} 


$$
\beta_{3}(z):=\frac{1}{2}\left[\beta_{2}(z)+(l+2)(l-1) \frac{j_{l}(z)}{z^{2}}\right] .
$$

The normalization constants $C(n, l)$ are given by $[16,22]$

$$
\begin{aligned}
|C(n, l)|^{2}= & \frac{4 \pi}{3}\left(k_{n l} R\right)^{3}\left\{\int _ { 0 } ^ { k _ { n l } R } \left[F_{1(n l)}(r)^{2}+l(l+1)\right.\right. \\
& \left.\left.\times F_{2(n l)}(r)^{2}\right] d\left(k_{n l} r\right)\right\}^{-1}
\end{aligned}
$$

where

$$
\begin{aligned}
F_{1(n l)}(r)= & \beta_{3}\left(k_{n l} R\right) k_{n l} r \frac{d}{d\left(q_{n l} r\right)} j_{l}\left(q_{n l} r\right)-l(l+1) \\
& \times \frac{q_{n l}}{k_{n l}} \beta_{1}\left(q_{n l} R\right) j_{l}\left(k_{n l} r\right) \\
F_{2(n l)}(r)= & \frac{k_{n l}}{q_{n l}} \beta_{3}\left(k_{n l} R\right) j_{l}\left(q_{n l} r\right) \\
& -\frac{q_{n l}}{k_{n l}} \beta_{1}\left(q_{n l} R\right) \frac{d}{d\left(k_{n l} r\right)}\left[k_{n l} r j_{l}\left(k_{n l} r\right)\right] .
\end{aligned}
$$

A more detailed exposition of the above computations can be found in [22].

Substituting Eq. (3.25) into Eq. (3.22) yields

$$
\begin{aligned}
P_{G W}= & \frac{12}{5 \pi \phi_{0} c^{5}} M^{2} R^{2}|C(n, 2)|^{2} \omega_{n 2}^{6}\left[\beta_{3}\left(k_{n 2} R\right) \frac{j_{2}\left(q_{n 2} R\right)}{q_{n 2} R}\right. \\
& \left.-3 \frac{q_{n 2}}{k_{n 2}} \beta_{1}\left(q_{n 2} R\right) \frac{j_{2}\left(k_{n 2} R\right)}{k_{n 2} R}\right]^{2}
\end{aligned}
$$

where $M$ is the total mass of the sphere. The oscillation energy of the five modes with $l=2$ is given by

$$
\begin{aligned}
E_{o s c}^{(n)}= & \frac{15}{2 \pi} M \omega_{n 2}^{2}|C(n, 2)|^{2} \frac{1}{\left(k_{n 2} R\right)^{3}} \int_{0}^{k_{n 2} R}\left\{F_{1(n 2)}(r)^{2}\right. \\
& \left.+6 F_{2(n 2)}(r)^{2}\right\} d\left(k_{n 2} r\right) .
\end{aligned}
$$

Making use of Eqs. (3.30) and (3.31), we can find the explicit value of Eq. (3.14) which, inserted into Eq. (3.21), leads to the final expression for the total cross section of a spin-two GW by a spherical detector in the context of JBD theory:

$$
\sigma_{t o t}^{(n)}=F_{n} \frac{G M v_{s}^{2}}{c^{3}} \frac{2 \omega_{B D}+3}{2\left(\omega_{B D}+2\right)} \frac{\Gamma}{\left(\omega-\omega_{n 2}\right)^{2}+\Gamma^{2} / 4},
$$

where

$$
v_{s}=\sqrt{2\left(1+\sigma_{P}\right)} \frac{\omega_{n l}}{k_{n l}}
$$

is the sound velocity,
TABLE I. Numerical values for $F_{n}$ and $H_{n}$.

\begin{tabular}{lll}
\hline \hline$n$ & \multicolumn{1}{c}{$F_{n}$} & \multicolumn{1}{c}{$H_{n}$} \\
\hline 1 & 2.98 & 1.14 \\
2 & 1.14 & 0.177 \\
3 & 0.110 & 0.0741 \\
4 & 0.0337 & 0.0408 \\
\hline \hline
\end{tabular}

$$
\sigma_{P}=\frac{\lambda}{2(\mu+\lambda)}
$$

is the Poisson ratio, and finally

$$
\begin{aligned}
F_{n}:= & \frac{2 \pi}{5\left(1+\sigma_{P}\right)}\left[\beta_{3}\left(k_{n 2} R\right) \frac{j_{2}\left(q_{n 2} R\right)}{q_{n 2} R}\right. \\
& \left.-3 \frac{q_{n 2}}{k_{n 2}} \beta_{1}\left(q_{n 2} R\right) \frac{j_{2}\left(k_{n 2} R\right)}{k_{n 2} R}\right]^{2}\left(k_{n 2} R\right)^{5} \\
& \times\left\{\int_{0}^{k_{n 2} R}\left[F_{1(n 2)}(r)^{2}+6 F_{2(n 2)}(r)^{2}\right] d\left(k_{n 2} r\right)\right\}^{-1} .
\end{aligned}
$$

It is useful to compute the integrated cross section

$$
\Sigma_{n}=\frac{1}{2 \pi} \int_{-\infty}^{+\infty} \sigma_{t o t}^{(n)} d \omega=\frac{G M v_{s}^{2}}{c^{3}} \frac{F_{n}\left(2 \omega_{B D}+3\right)}{2\left(\omega_{B D}+2\right)} .
$$

A few numerical values of $F_{n}$ are given in Table I for a standard value of the Poisson ratio, $\sigma_{P}=1 / 3$. For this value of $\sigma_{P}$, an analytic expression for the integral which appears in the definition of $F_{n}$ is given in the appendix. Note that Eq. (3.32) correctly reproduces the GR result $[13,23]$ in the limit $\omega_{B D} \rightarrow \infty$.

\section{B. Cross section for scalar GWs}

We now turn to a detailed computation of the cross section for a scalar GW. We begin by determining the energy flux of the incoming scalar waves, Eq. (3.10):

$$
\Phi_{(s)}=\hat{x}_{k}\left\langle t^{0 k}\right\rangle_{(s)}=\frac{\omega^{2} c^{4}}{8 \pi} \frac{2 \omega_{B D}+3}{\phi_{0}}|B|^{2},
$$

where the subscript $s$ stands for "scalar." If we denote the scattering amplitude by $W$, at large distances from the detector, and in complete analogy with Eq. (3.3) the scalar GW is a superposition of a plane and a scattered wave:

$$
\xi(\vec{x}, t) \rightarrow\left[B e^{i k \cdot x}+W(\hat{x}) \frac{e^{i \omega r}}{r}\right] e^{-i \omega t}+\text { c.c. }
$$

We can thus define incoming and outgoing amplitudes:

$$
\begin{aligned}
B^{\text {out }}(\vec{x}) & =\frac{1}{i \omega r}[B \delta(1-\hat{k} \cdot \hat{x})+i \omega W(\hat{x})], \\
B^{\text {in }}(\vec{x}) & =-\frac{1}{i \omega r} B \delta(1+\hat{k} \cdot \hat{x}),
\end{aligned}
$$


By substituting Eq. (3.39) into Eq. (3.37) one can compute the power associated with the outgoing part of the GW. The interference between the incident plane wave and the scattered wave leads to a contribution

$$
P_{i n t}=-\frac{\omega}{4 \pi \phi_{0}}\left(2 \omega_{B D}+3\right) \mathfrak{J}\left\{\int d \Omega \delta(1-\hat{k} \cdot \hat{x}) B^{*} W(\hat{x})\right\},
$$

while the contribution of the scattered wave is

$$
P_{\text {scat }}=\frac{\omega^{2}}{8 \pi \phi_{0}}\left(2 \omega_{B D}+3\right) \int d \Omega|W|^{2} .
$$

The scattering cross section is

$$
\sigma_{\text {scat }}:=\left(\frac{P_{\text {scat }}}{\Phi}\right)_{s}=\frac{\int d \Omega|W|^{2}}{|B|^{2}}
$$

and the total cross section is

$$
\sigma_{t o t}:=-\left(\frac{P_{i n t}}{\Phi}\right)_{s}=\frac{2}{\omega} \frac{\mathfrak{J}\left\{\int d \Omega \delta(1-\hat{k} \cdot \hat{x}) B^{*} W(\hat{x})\right\}}{|B|^{2}}
$$

The "quadrupole approximation" and the conservation of the stress-energy tensor imply $T_{i j}(\vec{k}, \omega)=\left(-\omega^{2} / 2\right) D_{i j}(\omega)$ and allow one to express the incoming wave amplitude as

$$
\begin{aligned}
B(\vec{x}, \omega) & \simeq-\frac{2}{\left(2 \omega_{B D}+3\right) r c^{4}} \int T\left(\vec{x}^{\prime}, \omega\right) e^{-i \vec{k} \cdot \vec{x}^{\prime}} d \vec{x}^{\prime} \\
& =-\frac{2}{\left(2 \omega_{B D}+3\right) r c^{4}} T(\vec{k}, \omega) \\
& =-\frac{2}{\left(2 \omega_{B D}+3\right) r c^{4}}\left[T_{j j}(\vec{k}, \omega)-T_{00}(\vec{k}, \omega)\right] \\
& =-\frac{2}{\left(2 \omega_{B D}+3\right) r c^{4}}\left[T_{j j}(\vec{k}, \omega)-\hat{x}_{j} \hat{x}_{k} T_{j k}(\vec{k}, \omega)\right] \\
& \simeq \frac{\omega^{2}}{\left(2 \omega_{B D}+3\right) r c^{4}}\left[\delta_{j k}-\hat{x}_{j} \hat{x}_{k}\right] D_{j k}(\omega) .
\end{aligned}
$$

In analogy with the form of Eq. (3.45), the scattering amplitude can be written as

$$
W(\hat{x})=\tau_{\beta}^{\beta}(\hat{x})=\left(\delta_{j k}-\hat{x}_{j} \hat{x}_{k}\right) \tau_{j k}
$$

and, once substituted into Eqs. (3.43), (3.44), yields

$$
\sigma_{\text {scat }}=\frac{8 \pi}{5} \frac{\left|\tau_{i i}\right|^{2}+\tau^{i j *} \tau_{i j} / 3}{|B|^{2}}
$$

$$
\sigma_{t o t}=\frac{4 \pi}{\omega} \frac{\mathfrak{J}\left\{B^{*}\left(\tau_{i i}-\hat{k}_{i} \hat{k}_{j} \tau_{i j}\right)\right\}}{|B|^{2}} .
$$

As in the spin-two case, the vibrational modes of the detector which are excited by an incoming GW depend on the polarization of the GW. Thus, in the case of a scalar GW propagating in the $+z$ direction, the excited modes are those with $\{l=m=0\}$ and also those with $\{l=2, m=0\}$. This is because the space components of the trace part of the GW tensor (2.10) must be expressed as a linear combination of $S_{(00)}^{j k}$ and $S_{(20)}^{j k}[16]$. For a spherically shaped detector, the eigenfrequencies corresponding to the spheroidal modes with quantum numbers $l=m=0\left(\omega_{0}\right)$ and $l=2, m=0\left(\omega_{2}\right)$ are numerically different $[13,16,17]$, and consequently we have to consider two cases: the scattering amplitudes for a $\mathrm{GW}$ travelling in the $z$-direction are given by

$$
\begin{aligned}
& \tau^{i j}(\omega)=\alpha B S_{(00)}^{i j} \text { for } \omega \simeq \omega_{0} \\
& \tau^{i j}(\omega)=\beta B S_{(20)}^{i j} \text { for } \omega \simeq \omega_{2},
\end{aligned}
$$

which, once substituted into Eqs. (3.47) and (3.48), in conjunction with Eq. (3.13), lead to the conditions

$$
\mathfrak{J}(\alpha)=\frac{2 \omega}{\eta_{0} \sqrt{4 \pi}}|\alpha|^{2}, \quad \mathfrak{J}(\beta)=-\frac{\omega}{2 \eta_{2} \sqrt{5 \pi}}|\beta|^{2} .
$$

At resonance $\left(\omega \simeq \omega_{2}\right.$ or $\omega \simeq \omega_{0}$ ) the modes of $T^{\mu \nu}$ behave as damped harmonic oscillators. Fourier transforming, one easily obtains the $\omega$-dependence of $\tau^{\mu \nu}$, and finally

$$
\begin{aligned}
& \alpha=\frac{\eta_{0} \sqrt{4 \pi}}{2 \omega}\left(\frac{-\Gamma_{0} / 2}{\omega-\omega_{0}+i \Gamma_{0} / 2}\right) \\
& \beta=\frac{2 \eta_{2} \sqrt{5 \pi}}{\omega}\left(\frac{\Gamma_{2} / 2}{\omega-\omega_{2}+i \Gamma_{2} / 2}\right) .
\end{aligned}
$$

The cross sections are thus given by

$$
\begin{aligned}
& \sigma_{t o t(00)}=\left(\frac{4 \pi \eta_{0} c^{2}}{\omega^{2}}\right) \frac{\Gamma_{0}^{2} / 4}{\left(\omega-\omega_{0}\right)^{2}+\Gamma_{0}^{2} / 4} \\
& \sigma_{t o t(20)}=\left(\frac{20 \pi \eta_{2} c^{2}}{\omega^{2}}\right) \frac{\Gamma_{2}^{2} / 4}{\left(\omega-\omega_{2}\right)^{2}+\Gamma_{2}^{2} / 4},
\end{aligned}
$$

where $\Gamma_{0}, \Gamma_{2}$ are the decay times of the free oscillation of the detector's modes with $l=0$ and $l=2$, and $\eta_{0}, \eta_{2}$ are defined as in Eq. (3.14). Note the geometrical ratio 5:1, related to the degeneracy of the quadrupole modes, between Eqs. (3.53) and (3.52) for a hypothetical detector with $\eta_{0}=\eta_{2}, \Gamma_{0}$ $=\Gamma_{2}$ and $\omega_{0}=\omega_{2}$.

The last thing which is left to do is the computation of the parameters $\eta_{0}, \eta_{2}$ in Eqs. (3.52),(3.53). Using Eq. (3.25) we find the power emitted by the detector due to the presence of the scalar field: 


$$
\begin{aligned}
P_{G W}= & \frac{\omega^{2} r^{2} c^{3}\left(2 \omega_{B D}+3\right)}{8 \pi \phi_{0}} \int|B(\omega)|^{2} d \Omega \\
= & \frac{\omega^{6}}{8 \pi \phi_{0}\left(2 \omega_{B D}+3\right) c^{5}} D^{i j *(\omega) D^{l m}(\omega)} \\
& \times \int\left(\delta_{i j}-\hat{x}_{i} \hat{x}_{j}\right)\left(\delta_{l m}-\hat{x}_{l} \hat{x}_{m}\right) d \Omega \\
= & \frac{\omega^{6}}{5 \phi_{0}\left(2 \omega_{B D}+3\right) c^{5}}\left[\left|D_{j j}(\omega)\right|^{2}+\frac{1}{3} D^{l m *}(\omega) D_{l m}(\omega)\right] \\
= & \frac{2}{5 \pi \phi_{0}\left(2 \omega_{B D}+3\right) c^{5}} M^{2} R^{2}\left(5 P_{00}+P_{20}\right),
\end{aligned}
$$

where

$$
\begin{aligned}
P_{00}= & |C(n, 0)|^{2} \omega_{n 0}^{6}\left[\beta_{3}\left(k_{n 0} R\right)\left(j_{2}\left(q_{n 0} R\right) / q_{n 0} R\right)\right]^{2} \\
P_{20}= & |C(n, 2)|^{2} \omega_{n 2}^{6}\left[\beta_{3}\left(k_{n 2} R\right)\left(j_{2}\left(q_{n 2} R\right) / q_{n 2} R\right)\right. \\
& \left.-3\left(q_{n 2} / k_{n 2}\right) \beta_{1}\left(q_{n 2} R\right)\left(j_{2}\left(k_{n 2} R\right) / k_{n 2} R\right)\right]^{2} .
\end{aligned}
$$

For the mode with quantum numbers $l=0, m=0$, putting together the first term in Eq. (3.54) and the oscillation energy

$$
\begin{aligned}
E_{o s c}^{(n 0)}= & \frac{3}{2 \pi} M^{2} \omega_{n 0}^{2} \frac{|C(n, 0)|^{2}}{\left(k_{n 0} R\right)^{3}} \beta_{3}\left(k_{n 0} R\right)^{2} \\
& \times \int_{0}^{k_{n 0} R}\left[k_{n 0} r j_{0}^{\prime}\left(q_{n 0} r\right)\right]^{2} d\left(k_{n 0} r\right),
\end{aligned}
$$

one finds

$$
\begin{aligned}
\eta_{0}= & \left(\frac{P_{G W}}{\Gamma_{0} E_{\text {osc }}}\right)_{(00)} \\
= & \frac{2 G M v_{t}^{2} \omega_{n 0}^{2}}{3 \Gamma_{0}\left(\omega_{B D}+2\right) c^{5}}\left[\left(j_{2}\left(q_{n 0} R\right) / q_{n 0} R\right)\right]^{2}\left(k_{n 0} R\right)^{5} \\
& \times\left\{\int_{0}^{k_{n 0} R}\left[k_{n 0} r j_{0}^{\prime}\left(q_{n 0} r\right)\right]^{2} d\left(k_{n 0} r\right)\right\}^{-1},
\end{aligned}
$$

where $v_{t}=\omega_{n l} / k_{n l}$. The total cross section for resonant scattering and absorption at $\omega \approx \omega_{n 0}$ of scalar GWs by a spherical detector is then

$$
\begin{aligned}
\sigma_{\text {tot }}^{(n 0)}= & \frac{2 \pi}{3} \frac{G M v_{t}^{2}}{c^{3}\left(\omega_{B D}+2\right)}\left[\left(j_{2}\left(q_{n 0} R\right) / q_{n 0} R\right)\right]^{2}\left(k_{n 0} R\right)^{5} \\
& \times\left\{\int_{0}^{k_{n 0} R}\left[k_{n 0} r j_{0}^{\prime}\left(q_{n 0} r\right)\right]^{2} d\left(k_{n 0} r\right)\right\}^{-1} \\
& \times \frac{\Gamma_{0}}{\left(\omega-\omega_{n 0}\right)^{2}+\Gamma_{0}^{2} / 4} \\
= & H_{n} \frac{G M v_{s}^{2}}{c^{3}\left(\omega_{B D}+2\right)} \frac{\Gamma_{0}}{\left(\omega-\omega_{n 0}\right)^{2}+\Gamma_{0}^{2} / 4},
\end{aligned}
$$

with

$$
\begin{aligned}
H_{n}:= & \frac{\pi}{3\left(1+\sigma_{P}\right)}\left[\left(j_{2}\left(q_{n 0} R\right) / q_{n 0} R\right)\right]^{2}\left(k_{n 0} R\right)^{5} \\
& \times\left\{\int_{0}^{k_{n 0} R}\left[k_{n 0} r j_{0}^{\prime}\left(q_{n 0} r\right)\right]^{2} d\left(k_{n 0} r\right)\right\}^{-1} \\
= & \frac{\pi}{3\left(1+\sigma_{P}\right)}\left[\left(j_{2}\left(q_{n 0} R\right) / q_{n 0} R\right)\right]^{2} \\
& \times\left(k_{n 0} R\right)^{2}\left(q_{n 0} R\right)^{3}\left[\frac{1}{2}\left(q_{n 0} R\right)+\frac{1}{4} \sin \left(2 q_{n 0} R\right)\right. \\
& \left.-\frac{\sin ^{2}\left(q_{n 0} R\right)}{q_{n 0} R}\right]^{-1} .
\end{aligned}
$$

Taking a standard value for the Poisson ratio, $\sigma_{P}=1 / 3$, we report in Table I the values of $H_{n}$ and $F_{n}$. It is useful to determine also the integrated cross section:

$$
\Sigma_{n 0}=\frac{1}{2 \pi} \int_{-\infty}^{+\infty} \sigma_{\text {tot }}^{(n 0)} d \omega=\frac{G M v_{s}^{2}}{c^{3}} \frac{H_{n}}{\omega_{B D}+2} .
$$

For the other mode, with quantum numbers $l=2, m=0$, using Eq. (3.31) and the second term in Eq. (3.54) one finds

$$
\begin{aligned}
\eta_{2}= & \left(\frac{P_{G W}}{\Gamma_{2} E_{o s c}}\right)_{(20)} \\
= & \frac{2 G M v_{t}^{2} \omega_{n 2}^{2}}{75 \Gamma_{2}\left(\omega_{B D}+2\right) c^{5}}\left[\beta_{3}\left(k_{n 2} R\right) \frac{j_{2}\left(q_{n 2} R\right)}{q_{n 2} R}\right. \\
& \left.-3 \frac{q_{n 2}}{k_{n 2}} \beta_{1}\left(q_{n 2} R\right) \frac{j_{2}\left(k_{n 2} R\right)}{k_{n 2} R}\right]^{2}\left(k_{n 2} R\right)^{5} \\
& \times\left\{\int_{0}^{k_{n 2} R}\left[F_{1(n 2)}(r)^{2}+6 F_{2(n 2)}(r)^{2}\right] d\left(k_{n 2} r\right)\right\}^{-1} .
\end{aligned}
$$

From this one gets the total cross section for resonant scattering and absorption at $\omega \approx \omega_{n 2}$ of scalar waves by a spherical detector:

$$
\begin{aligned}
\sigma_{t o t}^{(n 2)}= & \frac{2 \pi}{15} \frac{G M v_{t}^{2}}{c^{3}\left(\omega_{B D}+2\right)} \frac{\Gamma_{2}}{\left(\omega-\omega_{n 2}\right)^{2}+\Gamma_{2}^{2} / 4} \\
& \times\left[\beta_{3}\left(k_{n 2} R\right) \frac{j_{2}\left(q_{n 2} R\right)}{q_{n 2} R}\right. \\
& \left.-3 \frac{q_{n 2}}{k_{n 2}} \beta_{1}\left(q_{n 2} R\right) \frac{j_{2}\left(k_{n 2} R\right)}{k_{n 2} R}\right]^{2}\left(k_{n 2} R\right)^{5} \\
& \times\left\{\int_{0}^{k_{n 2} R}\left[F_{1(n 2)}(r)^{2}+6 F_{2(n 2)}(r)^{2}\right] d\left(k_{n 2} r\right)\right\} \\
= & \frac{F_{n}}{6} \frac{G M v_{s}^{2}}{c^{3}\left(\omega_{B D}+2\right)} \frac{\Gamma_{2}}{\left(\omega-\omega_{n 2}\right)^{2}+\Gamma_{2}^{2} / 4}
\end{aligned}
$$

where $F_{n}$ is given by Eq. (3.35). The corresponding integrated cross section is given by 


$$
\Sigma_{n 2}=\frac{1}{2 \pi} \int_{-\infty}^{+\infty} \sigma_{t o t}^{(n 2)} d \omega=\frac{G M v_{s}^{2}}{c^{3}} \frac{F_{n}}{6\left(\omega_{B D}+2\right)}
$$

From Table I we can infer the ratio between the integrated cross section for the modes with $l=0, m=0$, and the integrated cross section for the modes with $l=2, m=0$. For example for the vibrational mode with $n=1$, we find $\Sigma_{10} / \Sigma_{12}$ $=2.3$. As a last remark, we note that in the limit $\omega_{B D} \rightarrow \infty$ we recover GRs since $\sigma_{\text {tot }}^{(n 2)}, \sigma_{\text {tot }}^{(n 0)} \rightarrow 0$ and Eq. (3.32) tends to the value reported in [13]. The results of our calculations revise and extend some previous estimates of the cross sections obtained in [16], and will find an interesting application to a binary system of stars [24]. In the next section we will briefly consider the case of a burst of gravitational radiation emitted during the spherically symmetric collapse of a cloud of dust.

\section{DETECTABILITY OF SCALAR WAVE SIGNALS}

Let us now use the calculated cross sections to evaluate the detectability by a spherical detector of a possible scalar GW signal of astrophysical origin such as a burst from a gravitational collapse.

We consider the spherically symmetric collapse of a homogeneous dust ball (Oppenheimer-Snyder approximation), whose scalar GW emission and waveform have been recently studied [5]. The peak amplitude of the scalar GW in the JBD theory turns out to be

$$
b=\frac{\xi}{\phi_{0}} \simeq 10^{-23}\left(\frac{500}{\omega_{B D}}\right)\left(\frac{M_{*}}{M_{\odot}}\right)\left(\frac{10 \mathrm{Mpc}}{r}\right),
$$

where $M_{*}$ is the collapsing mass and $r$ is the distance from the source. The characteristic frequency $f_{c}$, defined as the frequency at which the energy spectrum of the waveform has its maximum value, is

$$
f_{c} \simeq 3 \times 10^{3}\left(\frac{M_{*}}{M_{\odot}}\right)^{1 / 2}\left(\frac{15 \mathrm{~km}}{r_{S}}\right)^{3 / 2} \mathrm{~Hz}
$$

where $r_{S}$ is the equatorial radius of the stellar surface before the collapse and is assumed to satisfy $r_{S}>4 M_{*} G c^{-2}$. Using the above figures, we can then estimate the possibility of detecting scalar GWs with a spherical detector. To this end it is convenient to define the energy absorbed by the detector's $n$th mode:

$$
\Delta E_{n}=\int_{0}^{\infty} \Phi(\omega) \sigma(\omega) d \omega \approx 2 \pi \Phi\left(\omega_{n}\right) \Sigma_{n}
$$

where $\Phi(\omega)$ is the incident GW energy flux per unit frequency. Using the above computed integrated cross sections one gets

$$
\Delta E_{n}=\frac{\pi K_{n} \Phi\left(\omega_{n}\right)}{\left(2+\omega_{B D}\right)} \frac{G M v_{s}^{2}}{c^{3}}
$$

where $K_{n}=2 H_{n}$ for the mode with $l=0$ and $K_{n}=F_{n} / 3$ for the mode with $l=2, m=0$. Using Eq. (3.37) with $b=B / \phi_{0}$ one finds

$$
\Delta E_{n}=\frac{1}{4} M v_{s}^{2}\left|b\left(\omega_{n}\right)\right|^{2} \omega_{n}^{2} K_{n}
$$

The detector's signal-to-noise ratio can be defined as

$$
\mathrm{SNR}=\frac{\Delta E_{n}}{\Delta E_{\min }}
$$

where $\Delta E_{\text {min }}$ is the minimum detectable energy innovation, depending on the detector's thermal and electronic noises. The theoretical bound on $\Delta E_{\text {min }}$ using linear readout systems is fixed by quantum mechanics to be $\hbar \omega_{n}$. For $\mathrm{SNR}=1$ one gets the minimum detectable value of the Fourier transform of the scalar GW amplitude:

$$
\left|b\left(\omega_{n}\right)\right|_{\min }=\left(\frac{4 \Delta E_{\min }}{M v_{s}^{2} \omega_{n}^{2} K_{n}}\right)^{1 / 2}
$$

As usual in the case of short bursts, i.e., bursts lasting for a time $\tau \approx 1 / f_{c}$ much shorter than the detector's characteristic damping time, the peak amplitude $b$ and the Fourier transform $b(\omega)$ at $\omega_{n}=2 \pi f_{c}$ can be related by $b \approx\left|b\left(\omega_{n}\right)\right| f_{c}$. The minimum detectable peak amplitude of the scalar $\mathrm{GW}$ is then

$$
|b|_{\min } \approx\left(\frac{\Delta E_{\min }}{\pi^{2} M v_{s}^{2} K_{n}}\right)^{1 / 2} .
$$

For instance let us consider a homogeneous spherical mass of a material with a high sound velocity such as molibdenum, recently added to the traditional list of materials used in GW research [25]. In order to have $\omega_{00} \approx 3 \mathrm{kHz}$, taken as a typical value in [5], with $v_{s}=5600 \mathrm{~m} / \mathrm{s}$, one has to take a detector diamater of $1.8 \mathrm{~m}$, and hence $M=31$ tons. Substituting into Eq. (4.8) we see that $b_{\min }=3 \times 10^{-22}$. From Eq. (4.1), and taking $\omega_{B D}=600$, we can estimate the maximum distance at which a solar mass collapse can be observed through the emission of scalar GWs to be $r_{\text {max }} \approx 0.3 \mathrm{Mpc}$. This range includes several galaxies in our Local Group. Assuming a rate of gravitational collapses of 1 event per 10 yr per galaxy, one may expect a resulting event rate approaching 1 event per $1 \mathrm{yr}$ in the detector.

\section{SUMMARY AND CONCLUSIONS}

Although Einstein's general theory of relativity is strongly supported by all experimental evidence available to date, certain alternative theories of the gravitational interaction naturally emerge out of more general theoretical schemes, notably string theory. It seems clear that any deviations from the predictions of general relativity must originate under very strong gravity conditions, such as stellar collapses. We naturally expect such phenomena to produce GWs, which will convey to the observer information both on the physics of the source and on the limits of a given theoretical model to understand that physics. One of the best known and well developed alternative theories to GR is Jordan-Brans-Dicke's scalar-tensor theory.

In this paper we have performed an in-depth analysis of how JBD gravitational waves interact with a spherical detector, which is particularly well suited to reveal or set thresh- 
olds on non-GR GW amplitudes, e.g., monopole amplitudes. This is a very specific feature of spherical detectors, for no other individual GW antenna constructed or conceived so far can possibly discriminate quadrupole from monopole $\mathrm{GW}$ radiation: an array of such detectors is required for this purpose, and this very significantly complicates detection techniques and algorithms.

We have expressed our results under the form of GW absorption cross sections for the different resonant modes of the antenna which get excited by those waves, and succeeded in finding closed analytic formulas for them. In particular, JBD waves excite the usual $m= \pm 2$ quadrupole modes of the spherical antenna, but they also excite the monopole mode and the $m=0$ quadrupole mode. Since the frequencies of these modes are different, we define suitable cross sections for the excitation of each of them.

Cross sections are of course very useful to define the sensitivity of a detector with a given level of noise: i.e., they enable an estimate of the signal-to-noise ratio. As a practical application, we have considered the signal emitted during the spherically symmetric collapse of a cloud of dusty matter-an event which would never occur should GR be the correct theory of gravity - and assessed the possibilities of seeing it with projected future spherical detectors. With the present bounds on the JBD parameter $\omega_{B D}$, we conclude that such events as this could be observed if they happen within our Local Group of galaxies, with an event rate of a rather encouraging one per year.

The possibility of sensing or thresholding monopole gravitational radiation with a single antenna is very promising, as it would contribute new and very important data to the understanding of the gravitational interaction, and also supply experimental evidence for sounder discussions of string theory.

\section{ACKNOWLEDGMENTS}

We would like to thank V. Fafone and V. Ferrari for useful discussions. M.B. and M.B. would like to thank C. N. Colacino for collaboration at the initial stages of this work. J.A.L. acknowledges financial support from the Spanish Ministry of Education, contract PB93-1050.

\section{APPENDIX}

In order to find an analytic expression for $F_{n}$ defined in Eq. (3.35) we have to perform the following integration:

$$
I_{n}=\int_{0}^{k_{n 2} R}\left[F_{1(n 2)}(r)^{2}+6 F_{2(n 2)}(r)^{2}\right] d\left(k_{n 2} r\right)
$$

where, in accordance with Eq. (3.29),

$$
\begin{aligned}
F_{1(n 2)}(r)= & \beta_{3}\left(k_{n 2} R\right) k_{n 2} r j_{2}^{\prime}\left(q_{n 2} r\right) \\
& -6 \frac{q_{n 2}}{k_{n 2}} \beta_{1}\left(q_{n 2} R\right) j_{2}\left(k_{n 2} r\right) \\
F_{2(n 2)}(r)= & \frac{k_{n 2}}{q_{n 2}} \beta_{3}\left(k_{n 2} R\right) j_{2}\left(q_{n 2} r\right) \\
& -\frac{q_{n 2}}{k_{n 2}} \beta_{1}\left(q_{n 2} R\right) \frac{d}{d\left(k_{n 2} r\right)}\left[k_{n 2} r j_{2}\left(k_{n 2} r\right)\right] .
\end{aligned}
$$

Since

$$
k_{n l}^{2}=q_{n l}^{2}\left(2+\frac{\lambda}{\mu}\right)
$$

choosing $\sigma_{P}=1 / 3$ yields $k_{n l}=2 q_{n l}$ and Eq. (A1) can be written in terms of the following integrals:

$$
\begin{aligned}
& G_{1}=\int_{0}^{k_{n 2} R} j_{2}(x)^{2} d x \\
& G_{2}=\int_{0}^{k_{n 2} R} x^{2} j_{2}^{\prime}(x)^{2} d x \\
& G_{3}=\int_{0}^{k_{n 2} R} x j_{2}^{\prime}(x) j_{2}(x) d x \\
& G_{4}=\int_{0}^{k_{n 2} R} x j_{2}^{\prime}(x) j_{2}(x / 2) d x \\
& G_{5}=\int_{0}^{k_{n 2} R} x j_{2}^{\prime}(x / 2) j_{2}(x) d x \\
& G_{6}=\int_{0}^{k_{n 2} R} j_{2}(x / 2) j_{2}(x) d x .
\end{aligned}
$$

Because $j_{2}(x)=\left(3 / x^{3}-1 / x\right) \sin x-\left(3 / x^{2}\right) \cos x$, we simply have to integrate by parts. For example, let us consider the first integral

$$
\begin{aligned}
G_{1}= & \int_{0}^{k_{n 2} R}\left[\left(9 / x^{6}+1 / x^{2}-6 / x^{4}\right) \sin ^{2} x+\left(9 / x^{4}\right) \cos ^{2} x-\left(6 / x^{2}\right)\left(3 / x^{3}-1 / x\right) \sin x \cos x\right] d x \\
= & \int_{0}^{k_{n 2} R}\left[9 / 2 x^{6}+1 / 2 x^{2}+3 / 2 x^{4}+\left(1 / 2 x^{2}\right)\left(15 / x^{2}-9 / x^{4}-1\right) \cos 2 x-\left(3 / x^{3}\right)\left(3 / x^{2}-1\right) \sin 2 x\right] d x \\
= & \frac{\operatorname{Si}\left(2 k_{n 2} R\right)}{5}-\frac{1}{2\left(k_{n 2} R\right)}-\frac{1}{2\left(k_{n 2} R\right)^{3}}-\frac{9}{10\left(k_{n 2} R\right)^{5}}+\frac{\cos \left(2 k_{n 2} R\right)}{10\left(k_{n 2} R\right)}-\frac{\sin \left(2 k_{n 2} R\right)}{5\left(k_{n 2} R\right)^{2}}-\frac{13 \cos \left(2 k_{n 2} R\right)}{10\left(k_{n 2} R\right)^{3}} \\
& +\frac{9 \sin \left(2 k_{n 2} R\right)}{5\left(k_{n 2} R\right)^{4}}+\frac{9 \cos \left(2 k_{n 2} R\right)}{10\left(k_{n 2} R\right)^{5}}
\end{aligned}
$$


where $\operatorname{Si}(x)=\int_{0}^{x}\left(\sin x^{\prime} / x^{\prime}\right) d x^{\prime}$. Solving in an analogous way all the other integrals, we finally obtain

$$
\begin{aligned}
I_{n}= & \frac{1}{8\left(k_{n 2} R\right)^{5}}\left\{3 \beta _ { 1 } ( q _ { n 2 } R ) ^ { 2 } \left[36 \cos \left(2 k_{n 2} R\right)+72 \sin \left(2 k_{n 2} R\right)\left(k_{n 2} R\right)-60 \cos \left(2 k_{n 2} R\right)\left(k_{n 2} R\right)^{2}-24 \sin \left(2 k_{n 2} R\right)\left(k_{n 2} R\right)^{3}\right.\right. \\
& \left.+6 \cos \left(2 k_{n 2} R\right)\left(k_{n 2} R\right)^{4}+\sin \left(2 k_{n 2} R\right)\left(k_{n 2} R\right)^{5}-36-12\left(k_{n 2} R\right)^{2}-6\left(k_{n 2} R\right)^{4}+2\left(k_{n 2} R\right)^{6}\right]+16 \beta_{3}\left(k_{n 2} R\right)^{2}\left[1728 \cos \left(k_{n 2} R\right)\right. \\
& +1728 \sin \left(k_{n 2} R\right)\left(k_{n 2} R\right)-720 \cos \left(k_{n 2} R\right)\left(k_{n 2} R\right)^{2}-144 \sin \left(k_{n 2} R\right)\left(k_{n 2} R\right)^{3}+16 \cos \left(k_{n 2} R\right)\left(k_{n 2} R\right)^{4}+\sin \left(k_{n 2} R\right)\left(k_{n 2} R\right)^{5} \\
& \left.-1728-144\left(k_{n 2} R\right)^{2}-16\left(k_{n 2} R\right)^{4}+\left(k_{n 2} R\right)^{6}\right]-96 \beta_{3}\left(k_{n 2} R\right) \beta_{1}\left(q_{n 2} R\right)\left[36 \cos \left(q_{n 2} R\right)+18 \sin \left(q_{n 2} R\right)\left(k_{n 2} R\right)\right. \\
& +3 \cos \left(q_{n 2} R\right)\left(k_{n 2} R\right)^{2}+3 \sin \left(q_{n 2} R\right)\left(k_{n 2} R\right)^{3}+\cos \left(q_{n 2} R\right)\left(k_{n 2} R\right)^{4}-36 \cos \left(3 q_{n 2} R\right)-54 \sin \left(3 q_{n 2} R\right)\left(k_{n 2} R\right) \\
& \left.\left.+33 \cos \left(3 q_{n 2} R\right)\left(k_{n 2} R\right)^{2}+9 \sin \left(3 q_{n 2} R\right)\left(k_{n 2} R\right)^{3}-\cos \left(3 q_{n 2} R\right)\left(k_{n 2} R\right)^{4}\right]\right\} .
\end{aligned}
$$

[1] P. Jordan, Z. Phys. 157, 112 (1959); C. Brans and R. H. Dicke, Phys. Rev. 124, 925 (1961).

[2] For a review see M. Green, J. H. Schwarz and E. Witten, Superstring Theory (Cambridge University Press, Cambridge, England, 1986).

[3] T. Damour and A. M. Polyakov, Nucl. Phys. B428, 331 (1994).

[4] M. Rees, R. Ruffini and J. A. Wheeler, Black Holes, Gravitational Waves and Cosmology: An Introduction to the Current Research (Gordon and Breach, New York, 1975); K. Thorne, in Three Hundred Years of Gravitation, edited by S. W. Hawking and W. Israel (Cambridge University Press, Cambridge, England, 1987); B. F. Schutz, in Gravitational Wave Experiments, Proceedings of the First Edoardo Amaldi Conference, Frascati, 1994, edited by E. Coccia, G. Pizzella and F. Ronga (World Scientific, Singapore, 1995).

[5] T. Harada, T. Chiba, K. Nakao and T. Nakamura, Phys. Rev. D 55, 2024 (1997).

[6] C. M. Will, Theory and Experiment in Gravitational Physics (Cambridge University Press, Cambridge, England, 1993).

[7] T. Damour and G. Esposito-Farèse, Phys. Rev. Lett. 70, 2220 (1993).

[8] G. Frossati and E. Coccia, Cryogenics 34, 9 (1994).

[9] E. Coccia, J. A. Lobo and J. A. Ortega, Phys. Rev. D 52, 3735 (1995)

[10] W. W. Johnson and S. M. Merkovitz, Phys. Rev. Lett. 70, 2367 (1993); Phys. Rev. D 51, 2546 (1995).
[11] P. F. Michelson and C. Z. Zhou, Phys. Rev. D 51, 2517 (1995).

[12] N. Ashby and J. Dreitlein, Phys. Rev. D 12, 336 (1975).

[13] R. V. Wagoner and H. J. Paik, in Proceedings of the International Symposium on Experimental Gravitation, Pavia, 1976 (Accademia Nazionale dei Lincei, Rome, 1977).

[14] M. Cerdonio et al., Phys. Rev. Lett. 71, 4107 (1993).

[15] M. Shibata, K. Nakao and T. Nakamura, Phys. Rev. D 50, 7304 (1994).

[16] J. A. Lobo, Phys. Rev. D 52, 591 (1995).

[17] M. Bianchi, E. Coccia, C. N. Colacino, V. Fafone and F. Fucito, Class. Quantum Grav. 13, 2865 (1996).

[18] J. Miller, M. Schneider, M. Soffel and H. Ruder, Astrophys. J. Lett. 382, L101 (1991).

[19] S. Weinberg, Gravitation and Cosmology (Wiley, New York, 1972).

[20] D. L. Lee, Phys. Rev. D 10, 2374 (1974).

[21] Handbook of Mathematical Functions, edited by M. Abramowitz and I. A. Stegun (Dover, New York, 1972).

[22] M. Brunetti, Studio Sulle Possibilità di Rivelare, Tramite un'Antenna Sferica, le Onde Gravitazionali Scalari e Tensoriali Emesse da Sistemi Binari (Tesi di Laurea, Università degli studi di Roma “La Sapienza," Anno Accademico 199596), unpublished.

[23] G. F. Torres de Castillo and L. C. Cortes Cuatli, Rev. Mex. Fis. 42, 550 (1996).

[24] M. Brunetti, E. Coccia, V. Fafone and F. Fucito (in preparation).

[25] W. Duffy et al., J. Appl. Phys. 81, 6675 (1997). 